\title{
Steering a simulated Unmanned Aerial Vehicle using a head-slaved camera and HMD: effects of HMD quality, visible vehicle references and extended stereo cueing
}

Sjoerd C. de Vries and Pieter Padmos

TNO Human Factors Research Institute, PO Box 23, 3769 ZG Soesterterg, Netherlands

\begin{abstract}
In the simulator experiment reported here we examined several parameters influencing the performance of the operator of a (simulated) Unmanned Aerial Vehicle (UAV). This operator was fitted with an HMD which showed images from the (virtual) camera onboard of the UAV. The camera was slaved to the operator's head so that the camera movements mimicked the head movements. We examined steering performance for two HMD types: the low-end, LCD-based Virtual $\mathrm{YO}$ i-glasses, and the high-end, CRT-based n-vision Dalavisor. Additional parameters were the presence of vehicle references in the images as an indication of camera orientation and the presence of stereo and byper-stereo (large baseline stereo). Performance withrthe n-vision HMD was considerably better than with the i-glasses HMD, a difference which could not be attributed solely to the hyper-stereo did not improve performance for this particular task.
\end{abstract}

Keywords: Head mounted displays, head slaved camera, Field of View (FOV), vehicle references, hyper-sterco, partial overlap.

\section{INTRODUCTION}

This paper reports the results of a study on the use of an HMD by the operator of a flying vehicle with a movable sensor platform controlled by the operator's head movements. This description not only covers situations where an operator is remotely controlling a small unmanned reconnaissance plane known as a UAV (Unmanned Acrial Vehicle) but also situations where an onboard pilot perceives his enviromment through the use of sensors, as can be the case in the Apache attack helicopter, where two independent sensor platforms consisting of an image intensifier and FLIR (Forward Looking InfraRed sensor) in the nose can be controlled by head-movements of pilot and gunner. In both cases, control of the sensor direction could have been accomplished manually. Intuitively, using bead-tracking appears to be a more ecological solution, because it enables the user to apply his vestibular and proprioceptive senses. Whetber or not this amangement really improves control performance is subject of future research. In this paper we concentrate on various parameters of the albovementioned set-up.

Grunwald, Kohn and Merhav' introduced research on the topic of 'line-of-sight slaving' in a flying task. However, their simulator study was mainly concemed with the perception of space by pilots using such a system. Flying performance was only compared with the performance using a large-FOV monitor that was not head slaved. The authors found a betler performance in the HMD condition. This performance advantage decreased when the pilots were required to make fixations at randomly appearing targets (not surprisingly since the task load increased). An extensive examination of line-of-sight-slaving related parameters was not the goal of their research.

Using an instrumented helicopter and various flying tasks, Kasper, Haworth, Szoboszlay, King and $\mathrm{Halmos}^{2}$ studied the effects. of various FOV restrictions on head movements and some flying performance measures. They found a clear influence of FOV on the head movements, though the patterns of differences were highly individual. The authors cite earlier research which shows that performance does not increase for FOVs above 80 degrees. Though restricted, the pilot's vision in this experiment was still natural and not intermediated by means of sensors and HMD. The study is therefore only a rough indication of the effecis the FOV of an HMD will have. Head-tracker lag. for instance, may be expected to affect head movements and therefore
performance. 
So and Griffin ${ }^{3}$ examined several head-coupled visual loops. Most of their research involves tracking and pursuit tasks, where a reticle visible in an HMD bas to be placed on a target by using head movements. So and Griffin found performance deteriorating above lags of $40 \mathrm{~ms}$. These particular head-coupled visual loops and tasks are not really the focus of our current rescarch.

In a previous simulator study, De Vries and Padmos ${ }^{4}$ examined the performance and head movements of subjects acting as UAV operators using a head slaved sensor and HMD feedback loop to perform their flying task. The parameters studied were: Field of View (FOV), sensor latency, the application of stereoscopy, and HMD-type. In order to vary the FOV, we simulated an HMD by using a projection dome where the application of a bead-slaved window of variable size allowed various amounts of the scene to be visible. Another HMD-simulation was made by projecting the whole scene on the dome, while the subjects wore FOV restricting goggles. In the sume experiment, performance and head movements of subjects using a Virtual- 10 i-glasses HMD was studied as well. The experiment involved flying along a wirding path marked by trees while keeping a constant height above an undulating ground level and a constant distance to the trees.

The results of this experiment showed a clear effect of FOV on performance, an effect possibly saturating beyond the 60 degrees. The decrease in performance due to an added sensor latency of $50 \mathrm{~ms}$ (on top of an image generating delay of about $70 \mathrm{~ms}$ ) was about half the decrease due to lowering the FOV from 57 degrees to 17 degrees. There was a small, though not significant tendency for latency to lower performance more for small FOVs than for large FOVs.

The use of simulated HMDs resulted in better performance than when the real HMD was used. This effect could not be attributed to resolution differences noe by vergence and accommodation issues. A difference between the two conditions was that the projection dome offered several possibilities for subject orientation, for instance because the three-channel projection system has clearly visible, narrow vertical overlapping areas. Those areas of non-uniform brightness were dome-fixed and hence vehicle-fixed, since the centre of the screen corresponded to the vehicle direction. It was hypothesized that adding visual references would improve performance and that the real HMD would benefit more from these vehicle references than the simulated HMD since the latter HMDs already had overlapping areas as references.

Stereopsis did not in any way improve performance, although the operator's task was to keep the UAV on at constant vertical distance to the ground $(3 \mathrm{~m})$ and a constant horizontal distance $(3 \mathrm{~m})$ to a series of identical trees marking a route. This distance of $3 \mathrm{~m}$ should be short enough to support stereo vision, normally. The ineffectiveness of stereopsis was explained in the study by assuming that the UAV operator needed to look far ahead, beyond the range where binocular vision is supported by the HMD. There may be alternatives to the 'planning ahead' hypotbesis, bowever. Furst, there were strong monocular cues: all trees were of the same size and their spacing was constant as well, which made stereo cues less relevant. Second, one could argue that the low quality optics of the system made the physical resolution of the HMD lower than the addressable resolution upon which our estimations of the maximum viewable depth were based. To test this we modificd the previous experiment in two ways: the tree lengths and spacings were randomized to reduce the amount of monocular cues, and to the monocular and stereo HMD condition we added a 'byper stereo' condition, where through doubling the inter-pupillary distance, the disparities were doubled. This would enlarge the perceivable depth range.

Additionally, we tried to replicate the abovementioned FOV study, not by using an head-tracked window of variable size as previously, but instead by using two FOV restricting goggles whose FOVs corresponded to the FOV of the virtual IO and a more sophisticated HMD (the n-vision Datavisor). Of course, the study also includes a comparison between performance of users wearing those HMDs. Since we can isolate the effects of FOV from effects related to other

\section{METHODS}

\subsection{Simulation hardware}

An Evans \& Sutherland ESIG 2000 high speed graphics processox was used to generate high resolution synthetic video images for the simulator vision system: 1500 to 2000 textured polygons set at $800 \times 600$ pixel resolution per channel. The image update frequency was $30 \mathrm{~Hz}$. Image presentation was either on a dome projection system (Seos PRODAS HiView S-600) with a maximal viewing area of $151 \times 45$ degrees of on an HMD (a Virtual 10 i-glasses or a n-vision Datavisor Hi Res, see figure 1) to display the images. The virtual IO HMD is a low-end LCD-based HMD with a FOV of $22 \mathrm{x} 18$ degrees (28 degrees diagonal) and NTSC video input (produced from the ESIG signal by an AverKey signal converter). The n-vision HMD is a high-end CRTbased display with a FOV of $60 \times 31$ degrees ( 67 degrees diagonal) when the Datavisor is used in a $50 \%$ overlap configuration 
(the Datavisor is also available in a $100 \%$ overlap configuration), which we used in SVGA mode ( $800 \times 600$ pixels). The subjects were seated in the centre of the projection dorne on a chair fitted with a joystick (Logitech Wingman). To register bead movements and to generate the appropriate visual feedback a sensor of a Pollolmus Fastrak magnetic head tracking system was mounted on the HMDs and on a light helmet worn by the subjects in the case of dome projection. The bead tracking delay amounted to about $15 \mathrm{~ms}$, the vehicle model calculations amounted to about $8 \mathrm{~ms}$, and the image generation cycle took about $70 \mathrm{~ms}$.
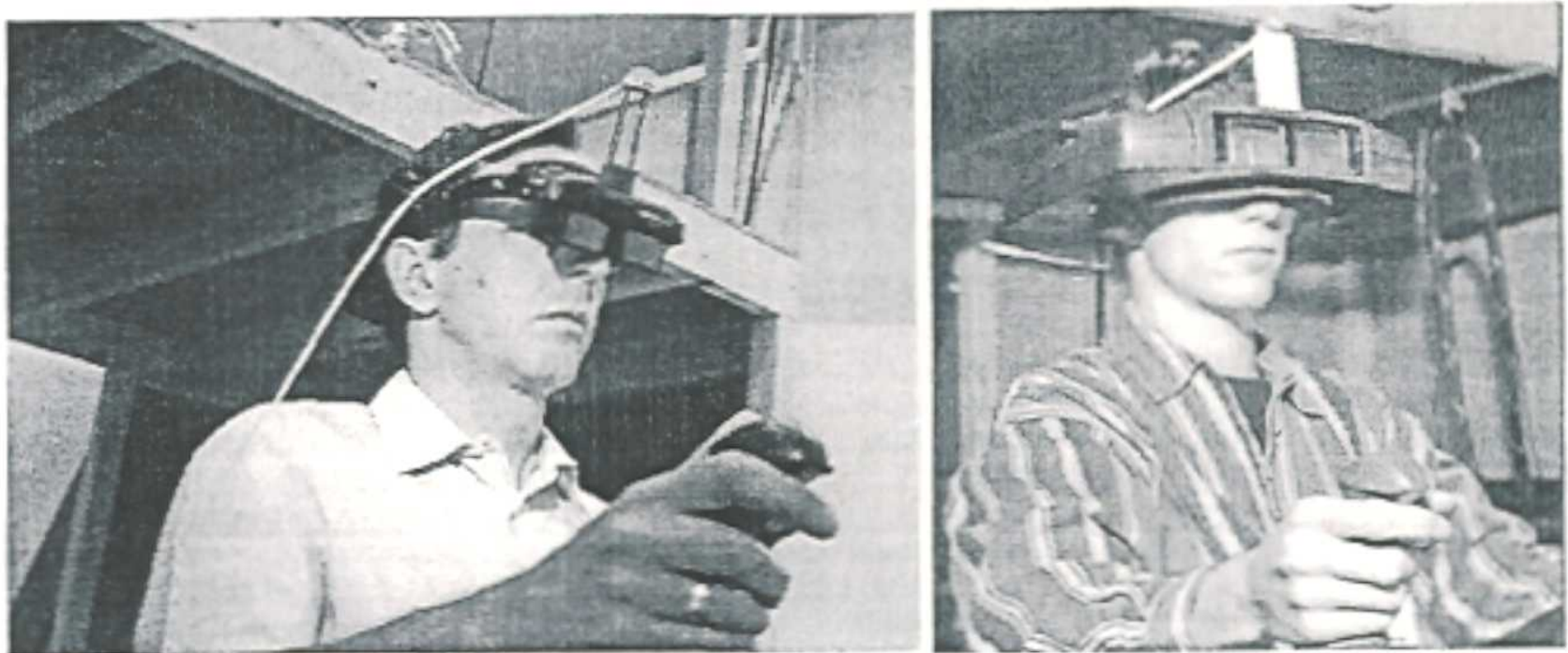

Figure 1. Two HMDs used in the experiment. The left-hand panel shows the Virtual IO i-glasses HMD with cover removed, the right hand panel shows the n-vision Datavisoc.

\subsection{Vehicle dynamic model}

A simple dynamic model was used for the UAV. The vehicle had a constant cruising speod of $25 \mathrm{~km} / \mathrm{h}$, and its yaw angle speed $(-2410+24 \% / \mathrm{s})$ and vertical speed $(-1$ to $+1 \mathrm{~m} / \mathrm{s})$ were controlled by movernents on the lateral and longitudinal axes of the joystick, respectively. To simulane the inertia of the vehicle the joystick signals were fed throagh a first order low pass filter with a time constant of $2 \mathrm{~s}$.

\subsection{Simulator database}

A set of 32 different routes was used. The routes consisted of successive curved segments, with right and left curves, marked with groups of trees of various sizes averaging $6 \mathrm{~m}$, spaced at irregular inter-tree distances, averaging $12 \mathrm{~m}$. The trees were alternately grouped in rod and green rows, with red trees being on the right side of the route and green trees on the left side. The terrain was textured and hilly. The route was limited on both sides by a dike, at $20 \mathrm{~m}$ from the centre of the route (see figure 2).

The shape of the path, including the slopes, was constructed by means of a parh generator, consisting of the UAV dynamic model controlled by time-varying simulated joystick input. The input signals bad a block shape which had passed a filter with a time constant of $2 \mathrm{~s}$. The heights of the signal blocks were drawn randomly from evenly distributed populations, with maxima equal to $80 \%$ of the maximum UAV yaw angle speed and vertical speed, respectively. This procedure guaranteed that it was physically possible to fly the route, whilst it was necessary to make bead movements to determine the path curvature, ẹven with the largest instantaneous field size. The signal block lengths were drawn randomly from an evenly distributed population, with a constraint that the path's direction should not cross itself. Each route started with a straight horizontal part of $75 \mathrm{~m}$. The length of each route was $1.7 \mathrm{~km}$. 


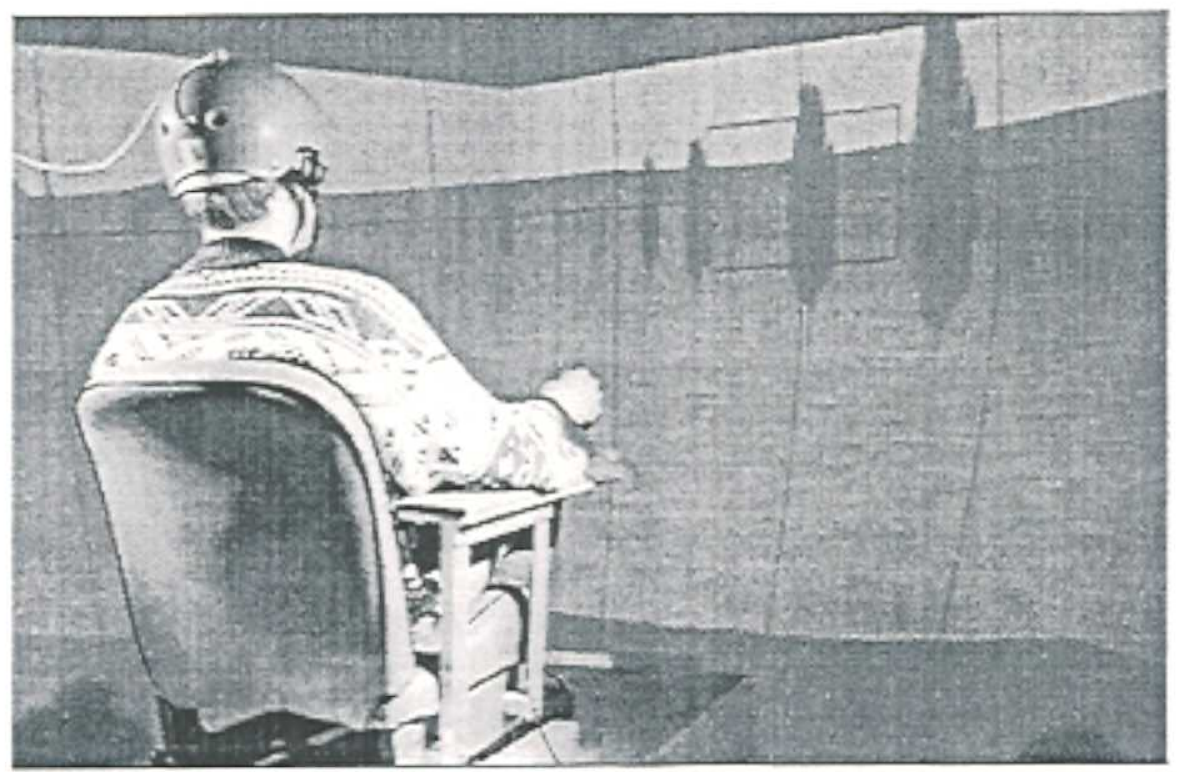

Figure 2. A view on the dome projection of one of the routes. Also visible are the vehicle references (window frames) used as one of the independent variables.

\subsection{Subjects, task, and training}

Eight paid subjects, male university students of age 18- 28 participated. They had nomal visual acuity, stereo acuity and colour vision, and were all right-handed. Subjects received a general instruction on aim and design of the experiment, followed by throe familiarization sessions. The subjects' task was to follow routes marked with trees, trying to keep a lateral distance of $4 \mathrm{~m}$ to the trees, and a height of $3 \mathrm{~m}$ above the ground. The red trees were to be kept on their right side, the green trees on their left side. The flying time of each route wis about 4 minutes.

In the training session subjects followed three different consecutive routes while they received both sound feedback and verbal feedhack on their performance. The continuous sound feedback consisted of tones provided by speakers to the left and the right of the subject, indicating horizontal and vertical crrors to the target path, in steps of $0.5 \mathrm{~m}$. Apart from the sounds, subjects received verbal feedback on performance and looking and stecring strategy from the instructorfsupervisos of the experiment. Training was given with an urrestricted FOV in the dome. Each experimental condition was also preceded by a training run with the corresponding visuals.

\subsection{Independent variables}

Four experimental factors were used in an incomplete factorial design:

\section{FOV}

Two FOV restricting goggles with FOVs corresponding to the i-glasses HMD and the nvision HMD (a FOV of 28 and 67 degrees, respectively) were used in combination with dome projection to simulate an HMD (see figure 3 ). The tubes on the goggles were positioned such that the left and right images owerlapped $100 \%$ at the projoction dome for the i-glasses simulation and $50 \%$ for the $\mathrm{n}$-vision simulation. When viewing through a real HMD the total viewable area was restricted to the sime areal which could be scanned on the projection dome. Based on the results of the previous experiment it was expected that the larger FOV would yicld better performance. 


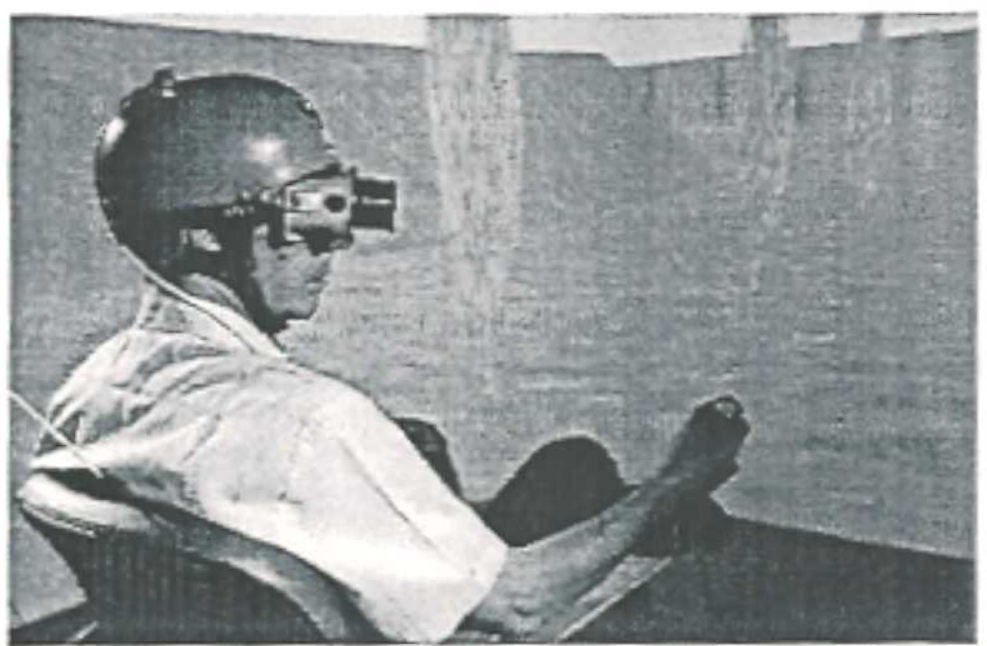

Figure 3. A picture of an HMD simulation. FOV restricting goggles are used in combination with a dome projection to simulate a head-tracked HMD.

\section{Vehicle references}

For two HMD-types, the real i-glasses HMD and the simulated i-glasses HMD, the presence of vehicle referenoes (see figure 2) was varied. A hypothesis derived from the previous experiment predicted that performance with the real HMD would show noticcable improvement and with the simulated HMD less or not at all dependent on how dominant the already existing vehicle references were in the simulated HMD case. In all other conditions vehicle references were present.

Stereo

The n-vision HMD was used in three modes: mono, stereo and hyper-stereo. In the latter case, the inter-pupillary distance used for the image calculations was doubled to enhance the visible depth-range.

\section{HMD-rype}

Four HMDs were utilized: the abovementioned simulated HMDs (two sizes) and the i-glasses and n-vision HMD. Because the two simulated HMDs have FOVs corresponding to the real HMDs this should allow a comparison of the $\mathrm{i}-\mathrm{glasses}$ HMD and the n-vision HMD corrected for FOV effects. If the differences between the two real HMDs are of the same magnitude as the differences between the two simulated HMDs then this effect may be altributed to FOV differences. However, if a significant interaction is found then the differences between the two HMDs are caused by other factors such as image quality, weight cte.

The eight viewing conditions making up the design are shown in table 1.

Table 1. An overview of the experimental factors and levels. The table entries marked with an $\mathrm{X}$ indicate the conditions that were carried out.

\begin{tabular}{|c|c|c|c|c|c|}
\hline & & \multicolumn{2}{|c|}{ FOV 28 degrees } & \multicolumn{2}{|c|}{ FOV 67 degrees } \\
\hline & & references & no references & references & no references \\
\hline Simulated HMD & mono & $\mathrm{X}$ & $\mathrm{X}$ & $\mathrm{X}$ & \\
\hline \multirow{3}{*}{ Real HMD } & mono & $\mathrm{X}$ & $\mathrm{X}$ & $\mathrm{X}$ & \\
\hline & stereo & & & $\mathrm{X}$ & . \\
\hline & byper-stereo & & & $\mathrm{X}$ & \\
\hline
\end{tabular}

\subsection{Dependent variables}

The dependent variables consisted of fying performance measures, head motion attributes, and subjective difficulty scores 
Flying performance. The following measures were derived from comparison of the UAV's position (sampled with $10 \mathrm{~Hz}$ ) with the target route (horizontal and vertical):

\author{
DISTH, DISTV \\ MDISTH, MDISTV \\ SDH, SDV
}

SDSPEEDH. SDSPEEDV the lateral orthogonal error and the beight error. relative to the target route (m)

the mean over one run of DISTH and DISTV (m), indicative for a bias in the errors.

the standard deviation over one run of DISTH and DISTV $(\mathrm{m})$, indicative for the size of the errors

the standard deviation over one run of lateral and vertical error speed (m/s), proportional to the difference in the errors of neighbouring samples, indicative for control effort.

Head motion attributes. From the head yaw and pitch data the following measures were derived:

SDYAW, SDPITCH the standard deviation over one run of yaw and pitch $\left({ }^{\circ}\right.$ )

STDYSP, STDPSP

the standard deviation over one run of yaw speed and pitch speed $\left({ }^{\circ} / \mathrm{s}\right)$

Subjective difficulty scores. After each viewing condition, the subject was asked to rate the subjective difficulty on a scale ranging from 1 - "(almost) no problem" to 5 - "(almost) unworkable".

\subsection{Procedure}

Each day of the experiment two subjects participated. They suocessively received, after the general training session (sect. 2.4), all eight viewing conditions, and flew a set of four routes per viewing condition, of which the first run was a training run with foedhock tones and verbal foedhack. Fach subject flew a toeal of 32 runs, in which all 32 different routes were presented. When one subject was flying a set of four runs, the other subject rested. The order in which viewing conditions were presented, as well as the order of routes was balanced across subjects; the eight sets of four routes were equally distributed over all eight viewing conditions so that all possible combinations of route sets and conditions occurred only once.

\subsection{Statistical processing}

Before analysis, outliers of the objective data set were removed and replaced with the corresponding group mean. This concerned $4 \%$ of the data. Analyses of variance were run with the package STATISTICA 5.0 ANOVAMANOVA, with the following sub-designs from the eight viewing conditions (seet. 2.5):

FOV

REFERENCES DISPLAY TYPE: STEREO: (small vs. large simalated HMD) $\times$ subject $(8)$

(Ref - no ref) $\times$ (i-glasses HMD vs. small simulated HMD) $\times$ subject(8)

(real HMD vs. Simulated HMD) $\times$ (small vs large FOV) $\times$ subject( 8 )

(HMD mono - HMD stereo - HMD hyper-stereo) $\times$ subject( 8 )

Significance of main effects and interactions was tested against interactions with the factor subject. The subjective difficulty ratings were transformed to $z$-seores before analysis of variance.

\section{RESULTS}

\subsection{Steering bias}

One may question whether the feedlock during the training runs suffices to learn to stay on a more or less invisible track. The results show that the average deviation from the perfect course was rather small, namely $0.1 \mathrm{~m}$ horizontally and $0.14 \mathrm{~m}$ vertically. There was no significant effect of viewing condition on this bias.

\subsection{Field Of View}

\section{Performance}

A first analysis did not show a significant effect of FOV, which contrasts with the results of the previous experiment. A plot of the individual performance shows one of the subjects to deviate strongly from the others (see figure 4). In the remainder of this section the data is analysed with the results of this subject removed. Besides this, the subject group was apparently considerably less homogenous as it was in the previous experiment. 

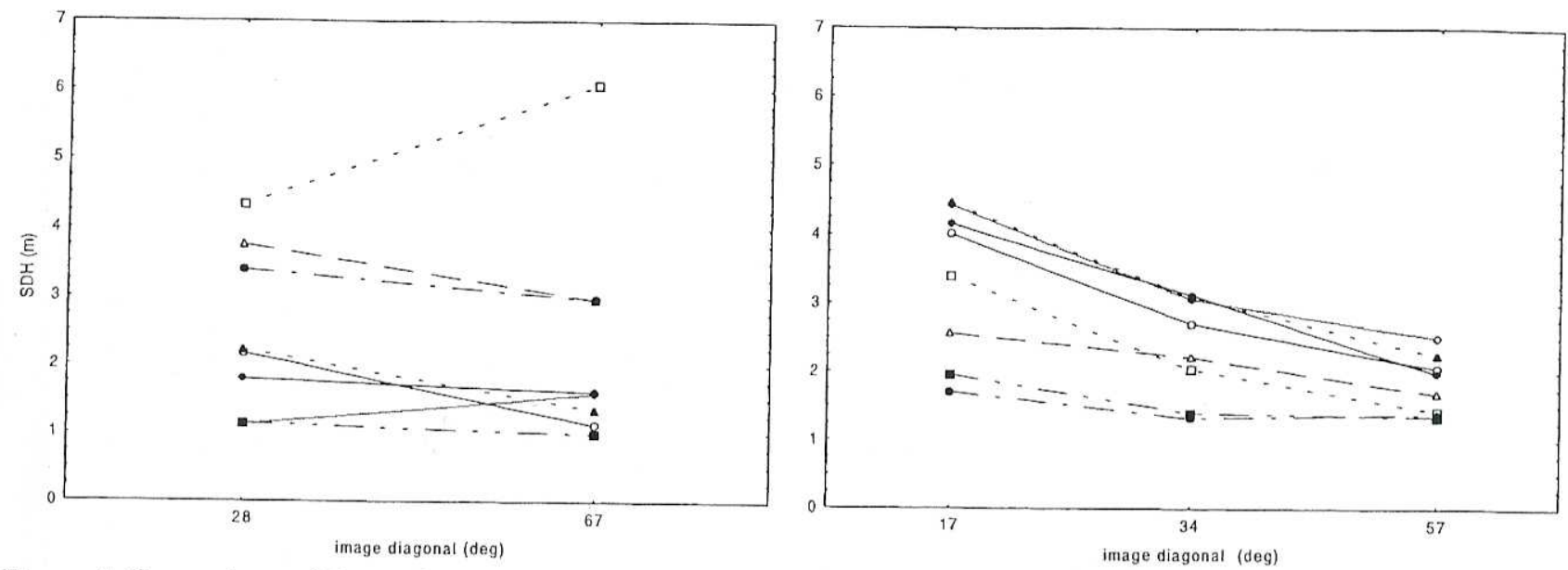

Figure 4. Comparison of the performance indicator SDH for the two FOV restrictors (left-hand panel) and the three sizes of head-tracked windows from the previous experiment (right-hand panel). Each line depicts the data of a single subject.

The data showed an increase in performance with increasing FOV (see Table 2), though only weakly for horizontal error indicators and not at all for vertical error indicators.

Table 2: Statistics for the effects of the factor FOV on steering performance.

\begin{tabular}{|l|l|l|l|l|}
\hline Performance indicator & small FOV & large VOF & $\mathrm{F}(1,6)$ & $\mathrm{p}$-level \\
\hline \hline standard deviation of the lateral error $(\mathrm{m})$ & 2.23 & 1.79 & 4.9 & $\mathrm{t}$ \\
standard deviation of the lateral error speed $(\mathrm{m} / \mathrm{s})$ & 0.69 & 0.61 & 8.1 & $*$ \\
standard deviation of the vertical error $(\mathrm{m})$ & 0.75 & 0.73 & 0.26 & $\mathrm{~ns}$ \\
standard deviation of the vertical error speed $(\mathrm{m} / \mathrm{s})$ & 0.23 & 0.23 & 0.03 & $\mathrm{~ns}$ \\
\hline \hline$* * * * \mathrm{p} \leq .0001 ; * * * \mathrm{p} \leq .001 ; * * \mathrm{p} \leq .01 ; * \mathrm{p} \leq .05 ; \mathrm{t} \mathrm{p}>.05 ; \mathrm{ns} \mathrm{p}>0.1$ & \\
\hline
\end{tabular}

\section{Head movements}

The FOV had no significant effect on most head movement descriptors (see Table 3). Like the performance data this contrasts clearly with the results of the previous experiment in which the subjects made less and slower movements for larger FOVs.

Table 3: Statistics for the effects of the factor FOV on head movement indicators.

\begin{tabular}{|l|l|l|l|l|}
\hline Head movement descriptor & small FOV & large VOF & $\mathrm{F}(1,6)$ & $\mathrm{p}$-level \\
\hline \hline standard deviation of the yaw (deg) & 11.0 & 12.1 & 2.5 & $\mathrm{~ns}$ \\
standard deviation of the yaw speed (deg/s) & 15.9 & 15.6 & 0.26 & $\mathrm{~ns}$ \\
standard deviation of the pitch (deg) & 2.6 & 2.7 & 0.16 & $\mathrm{~ns}$ \\
standard deviation of the pitch speed $(\mathrm{deg} / \mathrm{s})$ & 3.0 & 2.6 & 7.3 & $*$ \\
\hline \hline$* * * * \mathrm{p} \leq .0001 ; * * * \mathrm{p} \leq .001 ; * * \mathrm{p} \leq .01 ; * \mathrm{p} \leq .05 ; \mathrm{t} \mathrm{p}>.05 ; \mathrm{ns} \mathrm{p}>0.1$ & \\
\hline
\end{tabular}

\section{Subjective difficulty rating}

The subjective difficulty ratings were the only measurements which showed a clear difference between the two FOV values. The average ranking was 3.1 for the small (28 degrees diagonal) simulated HMD and 2.0 for the large (67 degrees diagonal) simulated $\mathrm{HMD}, \mathrm{F}(1,6)=117, \mathrm{p}=.00004$. 


\subsection{Vehicle references and HMD type}

This test was performed in order to see whether in our previous experiment differenoes between simulated and real HMDs were causcol by visible references on the projection dorre wich serve as vehicle references and therefore as an indication for vehicle heading. Since the simulated HMDs already had a kind of vebicle reference we would expect to see greatest improvement for the real HMD and hence a significant interaction between the factors vehicle references and HMD type.

\section{Performance}

Table 4 summarizes the performance dati. Clearly, both the effects of HMD-type and of wehicle references were modest, mostly between 10 and $20 \%$. The simulated HMD generally resulted in a better performance than the real HMD, but, except for one indicator, there was no significant difference between the HMD types. This seems to conflict with the results of our previous experiment. There is stronger evidence for an effect of vehicle references, although it is surprising to see that the strongest effect is for an indicator of vertical route following performance. Interaction between HMD-type and vehicle references is absent.

\section{Head movements}

Most head movement indicators showed a slight decrease in movements and off-centre positions of the head when vehicle references are added (see Table 5). Again, as found in De Vries and Padmos ${ }^{4}$ subjects wearing an Virtual IO HMD make less head movements and off-centre fixations than when they are wearing a simulated HMD. The low significance values for the pitch fixations can probably be explained from the restricted vertical FOV which causes vertical movements to be less useful.

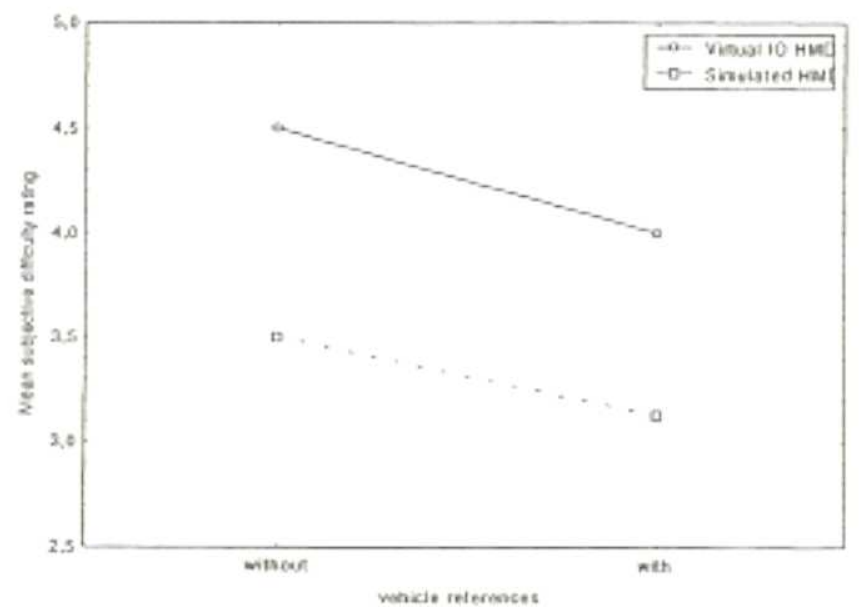

Figure 5. A comparison of the subjective difficulty with and without vehicle references for the Virtual IO HMD and the simulated HMD.

Subjective difficulty rating

The differences in subjective rating were more distinct than those found in the objective data. Figure 5 shows the effects of both HMD-type and vehicle references: the task was judged to be easier with the presence of vehicle references and using a simulated HMD. An ANOVA shows both main effects to be significant, $F(1,7)=8.3, p=0.02$ and $F(1,7)=7.4, p=0.03$ respectively. The effects of HMD-type and vehicle references dir not interact significantly. 
Table 4: Statistics for the effects of the factors Vehicle references and HMD type on steering performance.

\begin{tabular}{|c|c|c|c|c|c|c|}
\hline \multirow[b]{2}{*}{ Performance indicator } & \multicolumn{3}{|c|}{ MEANS } & \multicolumn{3}{|c|}{ SIGNIFICANCE } \\
\hline & & no ref & ref & factor & $\mathrm{F}(1,7)$ & p-level \\
\hline \multirow{3}{*}{$\begin{array}{l}\text { standard deviation of the } \\
\text { lateral crror (m) }\end{array}$} & virtual $10 \mathrm{HMD}$ & 3.3 & 3.1 & ref & 3.6 & $\overline{1}$ \\
\hline & simulated HMD & 2.9 & 2.5 & HMD & 2.4 & ns \\
\hline & & & & $\mathrm{ref} \times \mathrm{HMD}$ & 0.2 & ns \\
\hline \multirow{3}{*}{$\begin{array}{l}\text { standard deviation of the } \\
\text { lateral error speed }(\mathrm{m} / \mathrm{s})\end{array}$} & virtual IO HMD & 1.1 & 1.0 & ref & 10.3 & $*$ \\
\hline & simulated HMD & 0.9 & 0.7 & HMD & 4.6 & $t$ \\
\hline & & & & ref $\times \mathrm{HMD}$ & 0.6 & $\mathrm{~ns}$ \\
\hline \multirow{3}{*}{$\begin{array}{l}\text { standard deviation of the } \\
\text { vertical error (m) }\end{array}$} & virtual IO HMD & 1.1 & 0.9 & ref & 14.4 & 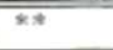 \\
\hline & simulated HMD & 0.9 & 0.8 & HMD & 17.0 & $* *$ \\
\hline & & & & ref $\times$ HMD & 2.2 & ns \\
\hline \multirow{3}{*}{$\begin{array}{l}\text { standard deviation of the } \\
\text { vertical error speed }\left(\mathrm{m}^{\prime} \mathrm{s}\right)\end{array}$} & virtual 10 HMD & 0.24 & 0.26 & $\mathrm{ref}$ & 0.2 & ns \\
\hline & simulated HMD & 0.24 & 0.23 & HMD & 2.0 & $\mathrm{~ns}$ \\
\hline & & & & $\mathrm{ref} \times \mathrm{HMD}$ & 2.3 & ns \\
\hline
\end{tabular}

Table 5: Statistic for the effects of the factors Vehicle references and HMD type on bead movement indicators.

\begin{tabular}{|c|c|c|c|c|c|c|}
\hline \multirow[b]{2}{*}{ Perfocmance indicator } & \multicolumn{3}{|c|}{ MEANS } & \multicolumn{3}{|c|}{ SIGNIFICANCE } \\
\hline & & no ref & ref & factor & $F(1,7)$ & p-level \\
\hline \multirow{3}{*}{$\begin{array}{l}\text { standard deviation of the } \\
\text { yaw (deg) }\end{array}$} & virtual IO HMD & 11.5 & 9.3 & ref & 36.9 & $2 x+4$ \\
\hline & simulated HMD & 14.3 & 11.0 & HMD & 12.5 & $*$ \\
\hline & & & & $\mathrm{ref} \times \mathrm{HMD}$ & 0.8 & ns \\
\hline \multirow{3}{*}{$\begin{array}{l}\text { standard deviation of the } \\
\text { yaw speed (deg/s) }\end{array}$} & virtual IO HMD & 9.7 & 9.0 & $\mathrm{ref}$ & 3.5 & $\mathrm{t}$ \\
\hline & simulated HMD & 16.6 & 15.0 & HMD & 10.3 & * \\
\hline & & & & ref $\times$ HMD & 0.6 & $\mathrm{~ns}$ \\
\hline \multirow{3}{*}{$\begin{array}{l}\text { standard deviation of the } \\
\text { pitch (deg) }\end{array}$} & virtual $10 \mathrm{HMD}$ & 2.7 & 2.4 & ref & 17.5 & *4 \\
\hline & simulated HMD & 2.7 & 2.5 & HMD & 0.1 & $\mathrm{~ns}$ \\
\hline & & & & ref $\times \mathrm{HMD}$ & 0.3 & ns \\
\hline \multirow{3}{*}{$\begin{array}{l}\text { standard deviation of the } \\
\text { pitch speed (deg/s) }\end{array}$} & virtual $10 \mathrm{HMD}$ & 2.6 & 2.7 & ref & 0.5 & $\mathrm{~ns}$ \\
\hline & simulated HMD & 3.3 & 2.9 & HMD & 2.7 & $\mathrm{~ns}$ \\
\hline & & & & ref $\times$ HMD & 15.5 & *** \\
\hline
\end{tabular}

\subsection{Stereo}

Although the experimental set-up was changed to increase the usefulness of the stereo cue the results did not show any significant change on any of the performance, head movement or subjective difficulty rating parameters.

\subsection{Comparison of the Virtual IO HMD and the n-vision HMD}

\section{Performance}

A direct comparison of both HMD types (with vehicle references present) showed a clear advantage of the n-vision HMD-(sce table 6). An especially large improvement was found for the performance in the horizontal plane. Comparing the performance differences between the two sizes of simulated HMDs and the two sizes of HMDs (figure fa) suggests that this difference is not due solely to the FOV differences between the Virtual IO and the n-vision HMD. 
Table 6. Comparison of performance with the virtual IO i-glasses HMD and the n-vision Datavisor

\begin{tabular}{|c|c|c|c|c|}
\hline Performance indicator & Virtual IO & n-vision & $\mathrm{F}(1.7)$ & $\mathrm{p}$-leve \\
\hline standard deviation of the lateral error (m) & 3.1 & 2.1 & 10.0 & $*$ \\
\hline standard deviation of the lateral error speed (m/s) & 0.9 & 0.8 & 10.6 & × \\
\hline standard deviation of the vertical error (m) & 1.0 & 0.7 & 15.4 & ** \\
\hline standard deviation of the vertical error speed (m/s) & 0.26 & 0.24 & 4.7 & 1 \\
\hline
\end{tabular}

\section{Head movements}

Some effects of HMD type on head movements were found (see table 7). Although the larger FOV of the a-vision HMD partially explains the atteauated head movements, an interaction graph of FOV and HMD type (figure Gb) shows that other differences between the Virtual IO and n-vision HMD must play a role. One factor may be the weight difference: the n-vision HMD wejghs $1.6 \mathrm{~kg}$, the Virtual IO only weighs $0.2 \mathrm{~kg}$. Please note that though the speceds may seem lowand the differences therein small, these values represent a large set of samples with fairly long periods of almost no movement at all, hence top speeds are several factors higher, and can reach a magnitude in the order of $200 \mathrm{deg} / \mathrm{s}$.

Table 7. Comparison of head movements with the virtual 10 i-glasses HMD and the n-vision Ditavisor

\begin{tabular}{|c|c|c|c|c|}
\hline Head movement descriptor & Virtual IO & n-vision & $\mathrm{F}(1.3)$ & p-level \\
\hline standard deviation of the yaw (deg) & 9.3 & 9.6 & 0.07 & ns. \\
\hline standard deviation of the yaw speed (degts) & 9.0 & 8.0 & 5.2 & $\mathrm{t}$ \\
\hline standard deviation of the pitch (deg) & 2.4 & 2.2 & 3.0 & ns \\
\hline standard deviation of the pitch speed (deg/s) & 2.7 & 1.9 & 6.5 & $*$ \\
\hline
\end{tabular}
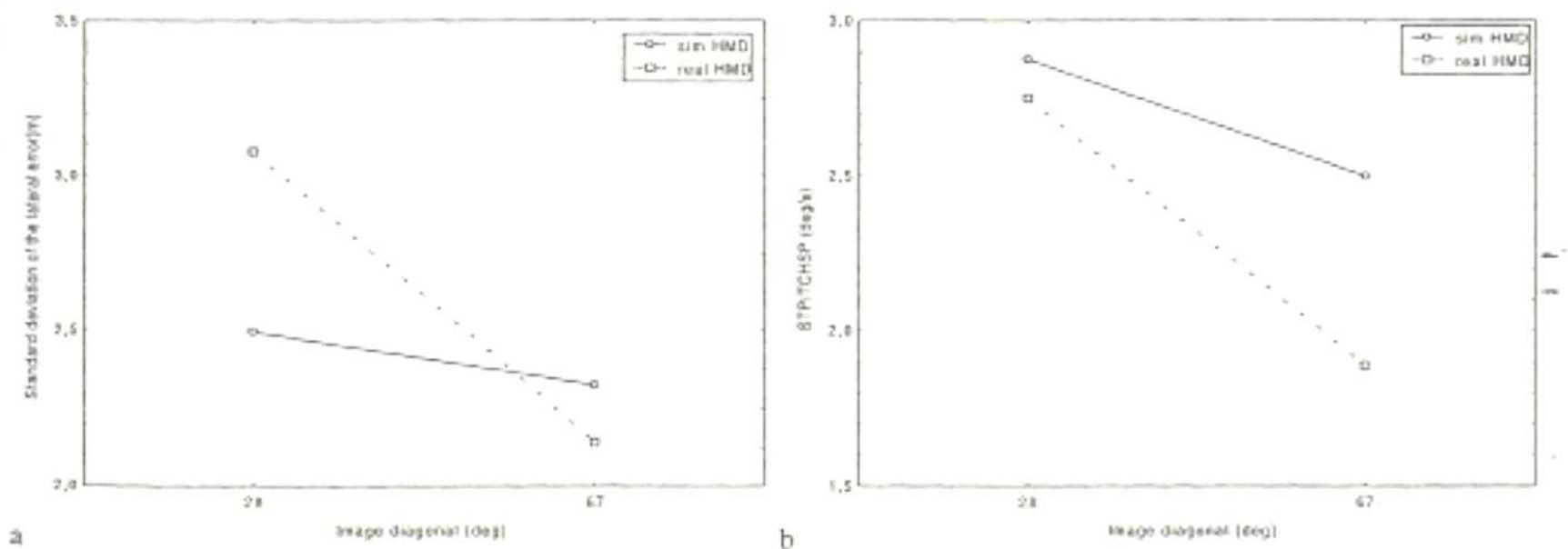

Figure 6. A comparison of the performance (a) and head movernents (b) using either a real or a simulated HMD, each in two sizes (where the small 'real HMD' is the Virnal IO HMD and the large 'real HMD' is the n-vision HMD). The interactions $(\mathrm{p}=0.008$ and $\mathrm{p}=0.05$, resp.) show that the difference between the two real HMDs can not be attributed solely to FOV differences. 
Subjective difficulty rating

Although the subjects complained considerably and consistently about the weight of the n-vision HMD their subjextive difficulty rating favoured this HMD ahove the i-glasses HMD, with a rating of 3.1 and 4.0 respectively, $F(1,7)=11,2, p=0.012$.

\section{CONCLUSIONS AND DISCUSSION}

We have measured the influence of Field Of View, HMD-type, vehicle references and stereoscopic presentation on performance, head movements and subjective difficulty ratings. Of these factors the largest effect was found for the HMD-type with a flight error increase of $50 \%$ if an i-glasses HMD is used instead of a n-vision HMD. The FOV was less a factor than in previous research. Vehicle references present in the imagery appeared to yicld a noticeable, but modest improvement. The addition of binocular sterco and even hyper-stereo did, despite more favourable conditions, not improve performance which reinforces our previous results.

\section{Field Of View}

Contrary to our previous research a clear effect of FOV on performance was not found. As noticed earlier this may be partly caused by subject effects. The individual behaviour is rather diverse, causing a low overall significance.

Another explanation may be found in the amount of bead movements. In our previous research we found a clear dependence of head movements on the FOV. If a head-slaved window is used to simulate an HMD, as it was in the previous experiment, the head tracker delay ${ }^{5}$ (ca. $15 \mathrm{~ms}$ ), the vehicle model computation delay (in the order of $8 \mathrm{~ms}$ ), and the image generator delay" (ca. $70 \mathrm{~ms}$ ) cause the window to lag behind the head direction by a distance of 0.7 degree (about the size of the fovea) at a head motion speed of $8 \mathrm{deg} / \mathrm{s}$ (typical) and 20 degrees at a speed of $200 \mathrm{deg} / \mathrm{s}$ (peak). The resulting instability may contribute to a lower performance at smaller FOVs. Since the FOV restriction of the simulated HMD in the current experiment was achieved without bead tracking there was no penalty in this sense for making head movements and perhaps therefore the effects of FOV were less pronounced.

A probable altemative may be that the advantage of a larger FOV of the n-vision simulation was countered by the disadvantage caused by the unnatural stimulus presented by the partial overlap sel-up of the n-vision HMD, a hypothesis for which there is some evidence? . For instance, partial overlap leads to visual illusions such as 'luning', an appcarance of a curved moon along the monocular border, where the binocular rivalry is strongest. Visual artefacts may also be the cause of the abovernentioned subject effects: some individuals may be more susceptible to interference caused by the partial overlap than others.

\section{Vehicle references}

Vehicle references generally improve the performance and the subjects' ratings indicated that references simplify the control task significantly. We did not find the predicted interaction between vehicle references and HMD type (i-glasses HMD and FOV restrictors) and therefore our hypothesis about the difference in performance in earlier experiments using the i-glasses HMD and the HMD simulations (the presence of implicit vehicle references in the latter) is falsified. Incidentally, the differenoe in performance between real and simulated HMDs in the current research is smaller than in the previous experiment. Again. this may be purely subject related.

\section{HMD type}

It was found that the i-glisses HMD yiclded a lower performance than the corresponding simulated HMD and that the n-vision yielded equal to or bether performance than the corresjoncling simulated HMD even though the ergonomics of the latter afpeatr to be sub-optimal in some aspects. This may indicate that image quality plays a role. Because the resolution differences cannot be an explanation ${ }^{24}$ a probable cause may be that the Virtual IO HMD is LCD based and the n-vision HMD is CRT based. ICDs are known to have motion smear problems and this may contribute to the lower performanoe. The human visual system is quite capable of compensating for motion blur occurring in its own optics ${ }^{11.12 .13 .14}$, but mily be unable to deal with visual input already blurred by motion. The fact that head movernents of Virtual-IO users are larger than those of n-vision users (probably due to weight and FOV differences) will increase the motion blur problem for the Virtual IO as well.

\section{Monoscopic presentation versus stereascopic presentation}

With the reproduction of the results of our previous research it seems soundly proven that for this type of control tasks stereopsis does nox offer any advantage. Because hyper-stereo increases the theoretical distance at which typical course deviations of $2 \mathrm{~m}$ 
can be noticed with the n-vision HMD from about 11 to $16 \mathrm{~m}$ (both of which are much higher than the required $3 \mathrm{~m}$ ), it can be surmised that the necessary 'planning distance' for this task exceeds the depth range that can be offered by this HMD for the applied inter-camera distance. Of course, this distance can be enlarged but that may increasingly distort the visual world and thereby induce additional steering errors. It is still possible that the other cues, especially the motion parallax cue, overwbelmthe stereo cue in importance for the execution of this task. The results do not indicate that stereo is not useful for other tasks.. Hovering and landing, which require manoeuvring in confined spaces, may be examples of tasks for which binocular stereo may prove to be a necessary condition.

\section{REFERENCES}

1. A.J. Grunwald, S. Kohn, and S.J. Merhav, "Visual field information in nap-of-the-earth flight by teleoperated helmetmounted displays", Proc of the SPIE conf. on Large-Serecn-Projection, Avionic, and Helmet-Mounted Displays, 1456, 132-153, 1991.

2. E.F. Kasper, L.A. Haworth, Z.P. Szobszlay, R.D. King and Z.L. Halmos, "Effects of in-flight field of view restriction on rotorcraft pilot head movement", Proc. of the SPIE conf. on Head Mounted Displays II, R.J. Lewandowski, L.A. Haworth and H.J. Girolamo (Eds,), 3058, 34-45.

3 R.H.Y. So, and M.J. Griffin, "Effect of lags on human performance with bead coupled simulators", Institute of Sound and Vibration Research, University of Southampton, report no. ISVR $92 / 33$ or Armstrong Liboratory, Wright-Patterson, report no. AL/CF-TR-1993-0101, 1993.

4. S.C. de Vries, and P. Padmos, Steering a simulated Unmanned Aerial Vehicle using a head-slaved camera and HMD. Proc. of the SPIE conff. on Head Mounted Displays II, R.J. Lewanckowski, L.A. Haworth and H.J. Girolamo (Eds.). 3058, 24-33 1997 .

5. B.D. Adelstein, E.R. Jobnston and S.R. Ellis, "Dynamic response of electromagnetic spatial displacement trackers", Presence, 5, 302-318, 1996.

6. J.H. Hogema, "Eflects of compensation for delay in the visual display of a driving simulator". TNO report IZF 1992 A-40, TNO Human Factors Research Institute, Socsterberg. The Netherlands, 1992

7. F. Kooi, "Perceptual aspects of partial binocular overlap in Head Mounted Displays; a literalture survey". TNO-report TM96-C003, TNO Human Factors Research Institute, Soesterberg, The Netherlands, 1996.

8. J.B.F. van Erp, "Effeets of update rate and spatial resolution on operator performance in a simulated unmanned ground vehicle". TNO-report TM-96-B008, TNO Human Factors Research Institute, Socsterberg, The Netherlands, 1996.

9. T. Kawara, M. Ohmi, and T. Yoshizawa, "Effects on visual functions during tasks of object handling in virtual environments with a head mounted display", Ergonomics, 39, 1370-1380, 1996.

11. R. Patterson and R. Fox, "Stereopsis during continuous head motion", Vision Research, 24, pp 2001-2003, 1984.

12 D. Regan, J.P. Frisby, G.P. Poggio, C.M. Schor and C.W. Tyler, "The perception of stereodepth and stereomotion". In Visual Perception, the neurophysiological foundations, L. Spillman and J.S. Werner (Eds.), pp. 317-347. Academic Press, Sin Diego, 1990.

13. G. Sperling, "Comparison of perception in the moving and stationary eye", In Eye movements an their role in visual and" cognitive processes, E. Kowler (Ed.). Elsevier Press, Amsterdam, 1990.

14. R.M. Steinman, and H. Collewein, "Binocular retinal image motion during active head rotation", Vision Research, 20, pp. $415-429,1980$. 